\title{
Cloning of novel soluble gp130 and detection of its neutralizing autoantibodies in rheumatoid arthritis
}

\author{
Masao Tanaka, ${ }^{1}$ Masaaki Kishimura, ${ }^{2}$ Shoichi Ozaki, ${ }^{1}$ Fumio Osakada, ${ }^{2}$ \\ Hidetaka Hashimoto, ${ }^{1}$ Mitsuo Okubo, ${ }^{3}$ Masao Murakami, ${ }^{1}$ and Kazuwa Nakao ${ }^{1}$ \\ ${ }^{1}$ Department of Medicine and Clinical Science, Kyoto University Graduate School of Medicine, Kyoto, Japan \\ ${ }^{2}$ Takasago Research Laboratories, Kaneka Corporation, Hyogo, Japan \\ ${ }^{3}$ Blood Transfusion Service, Saitama Medical Center, Saitama Medical School, Saitama, Japan
}

Address correspondence to: Shoichi Ozaki, Department of Medicine and Clinical Science, Kyoto University

Graduate School of Medicine, 54 Shogoin-Kawahara-cho, Sakyo-ku, Kyoto 606-8507, Japan.

Phone: 81-75-751-3204; Fax: 81-75-771-9452; E-mail: ozk@kuhp.kyoto-u.ac.jp.

Masao Tanaka and Masaaki Kishimura contributed equally to this work.

Received for publication June 2, 1999, and accepted in revised form May 31, 2000.

In an attempt to isolate disease-associated autoantigens in rheumatoid arthritis (RA), we cloned a new autoantigen named gp130-RAPS, which is a novel soluble form of the IL-6 signal-transducing molecule gp130. gp130-RAPS is a 50-kDa protein translated from alternatively spliced mRNA and has a truncated form of gp130 with a unique sequence, Asn-Ile-Ala-Ser-Phe (NIASF), in its COOHterminus. We observed serum antibodies to this NIASF sequence frequently in patients with RA, but not in those with other systemic rheumatic diseases or in healthy subjects. In RA, detection of those antibodies was significantly associated with disease activity indices such as serum C-reactive protein (CRP) levels, erythrocyte sedimentation rate, blood platelet counts, and serum IL-6 concentration. In vitro experiments revealed that gp130-RAPS inhibited IL-6 activity, and this inhibition was neutralized by antibodies to the COOH-terminus of gp130-RAPS derived from patients with RA. Thus, autoantibody to gp130-RAPS may play an important role in the progression of RA by promoting IL6 activity. Inspection of autoantibodies to gp130-RAPS may become a practical clinical test for RA. gp130-RAPS and its autoantibody provide a new clue to the complicated pathogenesis of RA.

J. Clin. Invest. 106:137-144 (2000).

\section{Introduction}

Rheumatoid arthritis (RA) is an autoimmune disease characterized by long-term inflammation and resultant destruction of multiple joints. In joint spaces of patients with RA, persistently presented antigens appear to play a major role in sustained inflammation by continuously stimulating $\mathrm{T}$ and $\mathrm{B}$ cells and thereby running cytokine cascades of TNF- $\alpha$, IL-1, IL- 6 , and so forth (1). Serum antibodies against the Fc portion of immunoglobulin $\mathrm{G}$ ( $\mathrm{IgG}$ ) molecules, known as rheumatoid factors (RFs), are important diagnostic markers, but not specific findings, because RFs are detected in some normal individuals and patients with various autoimmune diseases as well as in patients with RA (2). Autoantibodies or autoantigens specific to RA would serve as more useful indices for clinical evaluation of RA and would help in elucidating the pathogenesis of RA.

To find such disease-associated autoantigens in RA, we performed expression cloning of synovial antigens (3). As a result, we cloned new autoantigens, follistatinrelated protein (3) and a novel soluble form of gp130 described here. This soluble gp130 has a unique amino acid sequence, Asn-Ile-Ala-Ser-Phe (NIASF), in its $\mathrm{COOH}$-terminus. On the basis of the antigenicity of this $\mathrm{COOH}$-terminal sequence in RA, we named this novel protein gp130-RAPS (gp130 of the rheumatoid arthritis antigenic peptide-bearing soluble form), and its COOH-terminal 15-mer peptide RAPC15 (gp130RAPS $\mathrm{COOH}$-terminal 15 -mer peptide).

All tissues express gp130, and gp130 has a wide spectrum of biologic activity as a common signal transducer of IL-6 (4), leukemia inhibitory factor $(\operatorname{LIF})(5,6)$, oncostatin M (OSM) $(6,7)$, ciliary neurotrophic factor (CNTF) (5), IL-11 (8), and cardiotrophin-1 (CT-1) (9). Soluble gp130 (sgp130) lacking transmembrane and cytoplasmic regions was reported to inhibit the function of IL-6, OSM, LIF, and CNTF (10). Our cloned gp130-RAPS was also expected to have an inhibitory effect on such gp130-related cytokines.

Among the cytokines involved in the joint inflammation of RA, IL- 6 and its receptor components, IL- 6 receptor (IL-6R) and gp130, seem to play important roles in the activation of lymphocytes, synovial cells, and osteoclasts, not only in the production of pathogenic antibodies but also in the growth of synovial tissue and the destruction of joint structures $(4,11-14)$. Practically, IL-6 is abundantly released in synovial fluids and sera from patients with RA, and its serum concentration has a significant correlation with disease activity as evaluated by serum levels of CRPs (15). In addition, administration of anti-IL-6 or anti-IL-6R $m A b$ 's to patients with RA has been shown to exert beneficial effects in clinical trials $(16,17)$. 
In the present study, we demonstrate that gp130RAPS is an autoantigen in RA and has an inhibitory effect on IL- 6 and that autoantibodies to gp130-RAPS are specific to RA, correlate with disease activity, and block the IL-6-inhibitory function of gp130-RAPS.

\section{Methods}

Molecular cloning of gp130-RAPS. Details of our expression cloning method have been described previously (3).

Analysis of the gp130 genomic nucleotide sequence. Genomic DNA was obtained from PBMCs with a QIAamp Blood Kit (QIAGEN GmbH, Hilden, Germany). The 5' PCR primer was DF5P (5'-ATA CTG GAG TGA CTG GAG TG-3'), and the $3^{\prime}$ primer was DF3P (5'-CAT CTT GTG AGA GTC ACT TC-3'). They were located at nucleotides 924-943 and 1099-1118, respectively, in the gp130 cDNA (18). PCR was performed in $50-\mu \mathrm{L}$ reaction mixtures containing $15 \mathrm{pmol}$ primers, $500 \mathrm{ng}$ of genomic DNA, $200 \mu \mathrm{M}$ dNTPs, $0.5 \mu \mathrm{L}$ of a Perfect match PCR enhancer reagent (Stratagene Cloning Systems, La Jolla, California, USA), $0.5-\mu \mathrm{L}$ of LA-Taq polymerase (Takara Shuzo Co., Otsu, Japan), and buffer (preincubation at $94^{\circ} \mathrm{C}$ for 1 minute; 37 cycles of a three-step reaction at $98^{\circ} \mathrm{C}$ for 10 seconds, $50^{\circ} \mathrm{C}$ for 30 seconds, and $68^{\circ} \mathrm{C}$ for 5 minutes; and a final extension reaction at $72^{\circ} \mathrm{C}$ for 10 minutes). Aliquots of $50 \mathrm{ng}$ of purified PCR products were subjected to sequencing as described previously (3).

RT-PCR study of gp130-RAPS $m R N A$ expression. RNA was prepared from cultured cells with TRIzol reagent (GIBCO BRL, Gaithersburg, Maryland, USA). Doublestranded (ds) cDNA was synthesized from $5 \mu \mathrm{g}$ of total RNA using the cDNA Synthesis System kit (GIBCO BRL) and digested by Rsa I (Toyobo Co., Osaka, Japan) for 16 hours. PCR was performed in $50-\mu \mathrm{L}$ reaction mixtures containing $R s a$ I-digested ds cDNA from 50 ng of initial RNA, 15 pmol primers, $200 \mu \mathrm{M}$ dNTPs, 2 $\mathrm{mM} \mathrm{MgCl}_{2}$, and AmpliTaq Gold polymerase and GeneAmp PCR Gold buffer (Perkin-Elmer Applied Biosystems, Foster City, California, USA) (preincubation at $95^{\circ} \mathrm{C}$ for 9 minutes; $35-40$ cycles of a three-step reaction at $94^{\circ} \mathrm{C}$ for 30 seconds, $62^{\circ} \mathrm{C}$ for 15 seconds, and $72^{\circ} \mathrm{C}$ for 15 seconds; and a final extension reaction at $72^{\circ} \mathrm{C}$ for 5 minutes). In this PCR, the $5^{\prime}$ primer was DF5P2 (5'-GGA TAC TGG AGT GAC TGG AGT GAA G-3'; nucleotides 922-943), and the 3' primer was DF3P2 (5'-CCA TCT TGT GAG AGT CAC TTC ATA ATC-3'; nucleotides 1093-1119). The primer set for GAPDH transcripts was purchased from CLONTECH Laboratories Inc. (Palo Alto, California, USA).

IL-6 stimulation of synovial cells. The synovial cell line SF-1 was established from synovium derived from a patient with RA by a limiting dilution method. SF-1 cells have short processes and a round appearance. Their surface markers were as follows; CD14+, CD34-, HLA-DR ${ }^{+}, \mathrm{ICAM}^{+}$, and VCAM1-. They were free from infection with parvovirus or Epstein-Barr virus. Human recombinant IL-6 and soluble IL-6 receptor (sIL-6R) were purchased from R\&D Systems Inc. (Min- neapolis, Minnesota, USA). As synovial cells do not have IL-6R's or secrete sIL-6R, sIL-6R and IL-6 had to be added to the culture media in this experiment (13). IL-6 stimulation of SF-1 cells was performed as described previously (19).

Preparation of rabbit anti-RAPC15 antibody and its affinity columns. Rabbit anti-RAPC15 antibody ( $\alpha$-RAPC15 $\mathrm{Ab}$ ) was produced by immunization of ovalbumin conjugated with RAPC15 peptides by standard methods, and purified with a RAPC15-affinity column. The RAPC15-affinity column was prepared by coupling RAPC15 peptides with 2-fluoro-1-methylpyridinum toluene-4-sulfonate-activated cellulofine (Seikagaku Corp., Tokyo, Japan). $\alpha$-RAPC15 Ab-affinity columns were prepared by conjugating the affinity-purified rabbit $\alpha$-RAPC15 Ab with CNBr-activated Sepharose 4B (Amersham Pharmacia Biotech, Uppsala, Sweden).

Preparation of recombinant gp130-RAPS protein. Recombinant gP130-RAPS protein was purified from culture media of its cDNA-transfected COS-7 cells with the $\alpha$ RAPC15 Ab-affinity column. gp130-RAPS cDNA was ligated into PCXN2 vector and transfected into COS-7 cells using a CellPhect Transfection Kit (Amersham Pharmacia Biotech). pCXN2 vector was kindly provided by J. Miyazaki (Department of Nutrition, Osaka University, Osaka, Japan).

ELISA with antigen peptides. Each well of the microplates (Corning-Costar, Cambridge, Massachusetts, USA) was coated with $50 \mu \mathrm{L}$ of $20-\mu \mathrm{g} / \mathrm{mL}$ RAPC15 peptide in PBS overnight at $4^{\circ} \mathrm{C}$, blocked with $200 \mu \mathrm{L}$ of $5 \%$ BSA in PBS at room temperature for 2 hours, and reacted to $50 \mu \mathrm{L}$ of serum sample diluted $1: 40$ and 1:80, if necessary, and also 1:20 or 1:100-400 in PBS containing 5\% BSA in duplicate at room temperature for 2 hours. After three washes with PBS containing $0.05 \%$ Tween-20, bound antibodies were detected with $50 \mu \mathrm{L}$ of horseradish peroxidase-conjugated goat anti-human IgG antibody (Cappel, Durham, North Carolina, USA) diluted 1:2,000 in PBS containing 5\% BSA. Sample OD values were $O D$ values for antigen-coated wells minus those for uncoated wells. The titer (units per milliliter) of $\alpha$-RAPC15 Ab's was determined based on the standard curve for a positive control serum (the nondiluted serum was considered to contain $100 \mathrm{U} / \mathrm{mL}$ ).

Synthesis of antigen peptides. Peptides were synthesized with a Shimadzu PSSM-8 multiple peptide synthesizer (Shimadzu Corp., Kyoto, Japan) and purified to more than $90 \%$ by reverse-phase HPLC.

Fine epitope mapping. Diluted sera (1:200) were preincubated with competitive peptides $(10 \mu \mathrm{M})$ at room temperature for 30 minutes. The $\alpha$-RAPC15 Ab titers of these absorbed sera were measured by the ELISA system with RAPC15 peptides. The results are expressed as percent inhibition, i.e., percentages of the OD values for nonabsorbed sera minus those for absorbed sera over the OD values for nonabsorbed sera.

Evaluation of serum concentration of IL- 6 and RF. The serum concentration of IL- 6 was measured with an ELISA kit (Toyobo Co.). RF of the IgM class was meas- 
ured with an LX reagent Eiken RF-III (Eiken Kagaku, Tokyo, Japan).

Immunoprecipitation and immunoblotting of gp130-RAPS. gp130-RAPS in $1 \mathrm{~mL}$ of synovial fluid, plasma, culture medium concentrate, and cell lysate was immunoprecipitated by $2 \mu \mathrm{g}$ of anti-gp $130 \mathrm{mAb}$ GPX22 or $4 \mu \mathrm{g}$ of $\alpha$-RAPC15 Ab's conjugated with CNBr-activated Sepharose 4B. The characteristics of GPX22 have been reported previously (20). GPX22 was kindly provided by T. Taga (Tokyo Medical and Dental University, Tokyo, Japan). Elution of immunoprecipitates was performed as described elsewhere (3). The eluted samples $(10 \mu \mathrm{L})$ were subjected to SDS-PAGE under reducing conditions and immunoblotting with rabbit $\alpha$-RAPC15 $\mathrm{Ab}$ or goat anti-human gp130 antibody (AB-228-NA; R\&D Systems Inc.). Bound antibodies were detected with peroxidase-linked anti-rabbit Ig antibody (NA934; Amersham Pharmacia Biotech) or peroxidase-labeled antigoat IgG antibody (A201PS; American Qualex, San Clemente, California, USA).

Affinity purification of $\alpha$-RAPC15 Ab's. Three $\alpha$-RAPC15 $A b$ 's were purified from three patients with RA (RA1, RA4, and RA5) whose titers were $100,32.8$, and $6.3 \mathrm{U} / \mathrm{mL}$, respectively, with a HiTrap Protein G column (Amersham Pharmacia Biotech) and the RAPC15-affinity column. Control IgG was purified from a pooled serum mixture from six normal healthy subjects with the HiTrap Protein
G column. The deduced amounts of purified $\alpha$-RAPC15 Ab's in original sera from RA1, RA4, and RA5 were 52.3 (2.74\% in total IgG), 31.9 (1.86\% in total IgG), and 40.4 $\mu \mathrm{g} / \mathrm{mL}(1.89 \%$ in total $\operatorname{Ig} \mathrm{G})$, respectively.

Fibrinogen production assay. Human hepatoma cells Hep G2 (ATCC HB-8065; American Type Culture Collection, Rockville, Maryland, USA) in DMEM supplemented with $10 \%$ FCS were cultured in 96-well microtiter plates $\left(1 \times 10^{4}\right.$ cells per well, $0.2 \mathrm{~mL} /$ well $)$ with test samples for 24 hours $(20,21)$. Fibrinogen in culture supernatant under various experimental conditions was assayed by an FG-EIA ELISA kit (Enzyme Research Laboratories Inc., South Bend, Indiana, USA).

\section{Results}

Cloning and synovial expression of gp130-RAPS. To identify RA-specific autoantigens, we screened synovial-cell cDNA expression libraries with IgG probes from patients with RA, and we isolated several cDNAs (3). One of these cDNAs encoded a novel soluble form of gp130, gp130-RAPS (DDBJ/EMBL/GenBank accession number: AB015706). It had the nucleotide sequence of gp130 (-91 to 1869) lacking an 83-bp fragment (974 to 1056) (18), and a new stop codon located at about $5^{\prime}$ one third of the original coding region due to a frame shift. As a result, gp130-RAPS had NIASF in its $\mathrm{COOH}$ terminus, lacking transmembrane and cytoplasmic por- a

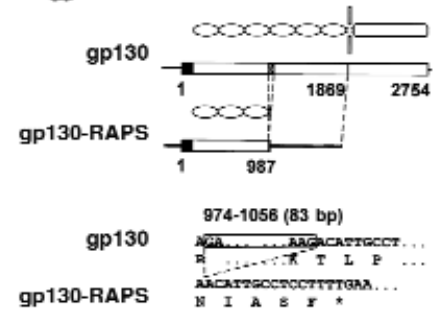

b

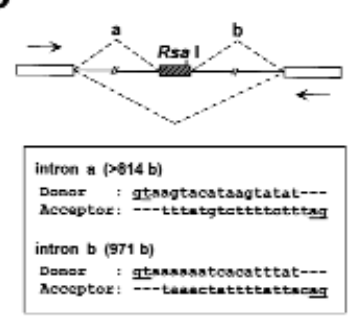

C

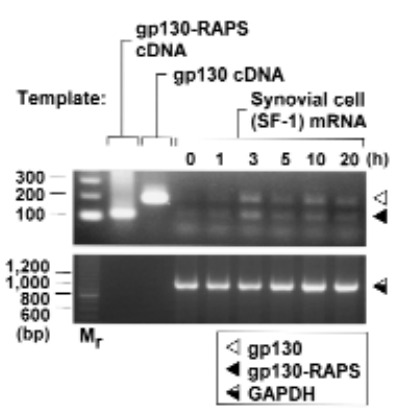

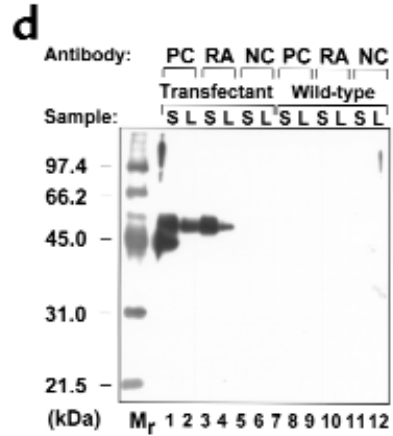

Figure 1

Schematic representations of gp130-RAPS cDNA and its partial genome structure (a and $\mathbf{b}$ ), as well as a demonstration of its mRNA expression (c) and translated products (d). (a) Top: An 83-bp fragment (hatched) was deleted in gp130-RAPS cDNA. Each gp130-RAPS molecule had three intact fibronectin type III modules (ovals) and lacked a cytoplasmic region (rounded rectangle). Bottom: The 83-bp deletion brought about a frame shift and addition of the unique amino acid sequence NIASF followed by a stop codon. Nucleotide numbers are the same in the membrane-bound gp130 (18). (b) The gp130 genomic sequence had at least two introns adjacent to the deleted 83-bp exon fragment (hatched) in gp130-RAPS. Each intron had a donor-acceptor structure compatible with the GT-AG rule (shown in the box, underlined nucleotides). It seemed that in mRNA splicing, loss of introns a and b, together with the 83-bp exon, produced gP130-RAPS mRNA, and that their loss kept that exon preserved and created gp130 mRNA. Arrows indicate primers for sequencing and RT-PCR. An Rsa I site was located in the 83-bp exon. (c) RT-PCR analysis demonstrated gp130-RAPS mRNA expression in IL-6-stimulated SF-1 synovial cells. The expression was upregulated after 3-hour stimulation by IL-6 $(100 \mathrm{ng} / \mathrm{mL})$ with slL-6R $(100 \mathrm{ng} / \mathrm{mL})$. PCR was performed for up to $35 \mathrm{cycles}$. In 40-cycle PCR, the bands of gp130-RAPS differed little in density (data not shown). As shown in the control lanes, 115-bp and 198-bp bands represent the products of gp130-RAPS and gp130 mRNA, respectively. GAPDH gene expression, represented by 983-bp bands, was used as a control to ensure equivalent amounts of original templates. Rsa I digestion could not completely eliminate nonspliced gP130 mRNA derivatives. (d) Rabbit $\alpha$-RAPC15 Ab (positive control, PC) and RA patient (RA1) $\alpha$-RAPC15 Ab (RA) reacted to gP130-RAPS and immunoprecipitated it mainly from culture supernatant (S) of gp130-RAPS cDNA-transfected COS-7 cells (transfectant) (lanes 1 and 3 ), and a little from their lysate (L) (lanes 2 and 4). gp130-RAPS protein had an approximate molecular weight of $50 \mathrm{kDa}$ under reducing conditions. An extra band with a lower molecular weight was probably formed by partially degraded molecules (lane 1 ). IgG from normal healthy subjects (negative control, NC) did not react to gp130-RAPS (lanes 5 and 6). Sample controls derived from nontransfected COS-7 cells were also examined (wild-type) (lanes 7-12). Immunoblotting was performed with rabbit $\alpha$-RAPC15 Ab. 
Table 1

Association of anti-RAPC15 Ab positivity with disease activity parameters in patients with RA

\begin{tabular}{|c|c|c|c|c|}
\hline \multirow[b]{2}{*}{ Parameter } & \multicolumn{2}{|c|}{ Anti-RAPC15 antibody } & \multicolumn{2}{|c|}{$P$} \\
\hline & $\begin{array}{l}\text { Positive }^{\mathrm{A}} \\
(n=54)\end{array}$ & $\begin{array}{l}\text { Negative }^{\mathrm{A}} \\
(n=20)\end{array}$ & $U$ test $^{\mathrm{B}}$ & $t$ test $^{\mathrm{C}}$ \\
\hline $\mathrm{CRP}(\mathrm{mg} / \mathrm{dL})$ & $2.7 \pm 2.4$ & $1.1 \pm 1.6$ & $<0.001$ & $<0.01$ \\
\hline $\operatorname{ESR}(\mathrm{mm} / \mathrm{h})$ & $50.3 \pm 29.8$ & $26.1 \pm 18.3$ & $<0.001$ & $<0.005$ \\
\hline Platelets $\left(\times 10^{-3} / \mu \mathrm{L}\right.$ & $316.4 \pm 93.6$ & $251.1 \pm 62.0$ & $<0.005$ & $<0.01$ \\
\hline $\mathrm{IL}-6(\mathrm{pg} / \mathrm{mL})$ & $24.1 \pm 18.9$ & $12.1 \pm 11.5$ & $<0.005$ & $<0.05$ \\
\hline $\mathrm{RF}(\mathrm{U} / \mathrm{mL})$ & $190.7 \pm 318.2$ & $126.2 \pm 186.7$ & NS & NS \\
\hline
\end{tabular}

${ }^{A}$ Values are means $\pm S D$. ${ }^{B}$ Mann-Whitney's $U$ test. 'Student's $t$ test.

tions (Figure 1a). We have not determined the $3^{\prime}$ sequence of gp130-RAPS cDNA from nucleotide 1869 to the poly A tail.

To determine how gp130-RAPS mRNA is generated and to confirm whether it is actually produced, we examined the exon-intron structure in the genomic gp130 DNA and analyzed the manner of gp130-RAPS mRNA expression in synovial cells. Sequencing of a part of the genome with a PCR method indicated that there were at least two introns with a donor-acceptor structure in the gene (Figure 1b; DDBJ/EMBL/GenBank accession number: intron $\mathrm{a}, \mathrm{AB} 015703$ and $\mathrm{AB} 015704$; intron $\mathrm{b}$, $\mathrm{AB} 015705)$. There were no differences among the sequences determined from four individuals (two were patients with RA, and two were healthy controls). This result suggested that two mRNA sequences can be produced by alternative splicing. The longer mRNA was suspected to encode gp130, and the shorter one (83bp-spliced fragment), to encode gp130-RAPS. To examine the expression of gp130-RAPS mRNA, we used a RT-PCR method with the following modifications. To reduce competitive amplification of the longer sequence, which overcame that of the shorter sequence for some reason, e.g., the relative quantity of the template or its structure favorable to PCR, we digested cDNAs at the Rsa I site to eliminate the longer sequence (Figure 1b). We actually found gp130-RAPS mRNA expressed in synovial cells (SF-1 cells), and its expression was upregulated by IL-6 with sIL-6R after 3-hour stimulation (Figure 1c).

In SF-1 cells, other gp130-related cytokines, OSM and LIF, also upregulated gp130-RAPS mRNA expression. Hep G2 cells also showed gp130-RAPS mRNA expression that was augmented by IL-6, OSM, and LIF. We detected gp130-RAPS mRNA in concanavalin A-treated PBMCs, but hardly at all in nontreated ones. The data are not shown here.

To examine whether gp130-RAPS mRNA is actually translated and expressed, and whether $\alpha$-RAPC15 Ab's react to gp130-RAPS molecules, we transferred gp130RAPS cDNA into COS-7 cells and immunoprecipitated the translated products with $\alpha$-RAPC15 Ab's from culture media and cell lysates. Both rabbit antiserum and $\alpha$ RAPC15 Ab's from patients with RA predominantly reacted with gp130-RAPS expressed in culture media and identified it as a secreted 50-kDa protein (Figure 1d).

Epitopes of gp130-RAPS. As the COOH-terminal NIASF sequence of gp130-RAPS is unique and not present in gp130, we thought that the epitopes of the gp130RAPS molecule might be located in this sequence, and we performed ELISA with the antigen peptide RAPC15 and various competitor peptides (Figure 2). The $\mathrm{COOH}$-terminal 10-mer peptide in RAPC15 (peptide 2) showed an inhibitory effect identical to that of RAPC15 (peptide 1, control). The 10-mer peptide lacking $\mathrm{COOH}$-terminal phenylalanine (peptide 3 ) and the one in which either isoleucine 326 or COOH-terminal phenylalanine 329 was replaced by glycine (peptides 5 and 8) exhibited no significant inhibition (<25\%). However, the 10-mer peptides in which one amino acid residue except isoleucine 326 and phenylalanine 329 in the NIASF sequence was replaced by glycine (peptides 4,6 , and 7 ) showed considerable inhibition (> $75 \%$ for two serum samples). Thus, isoleucine 326 and $\mathrm{COOH}-$ terminal free phenylalanine 329 , both of which are hydrophobic, appeared to contribute greatly to the antigenicity of RAPC15. The glycine 5-mer peptide bearing the $\mathrm{COOH}$-terminal NIASF sequence (peptide 9) did not show complete inhibition. The glycine 9-mer peptide bearing $\mathrm{COOH}$-terminal phenylalanine (peptide 10) demonstrated little inhibition, showing that the $\mathrm{COOH}$-terminal free aromatic amino acid phenylalanine was not antigenic in itself. These results suggested that the binding of antibodies to RAPC15 peptides required the $\mathrm{COOH}$-terminal NIASF sequence together with at most five additional amino acid residues to its $\mathrm{NH}_{2}$-terminus and that isoleucine and phenylalanine are indispensable.

Prevalence of $\alpha-R A P C 15 A b$ 's in systemic rheumatic diseases and relation of the antibodies with disease activity in $R A$. To investigate the prevalence of antibodies to

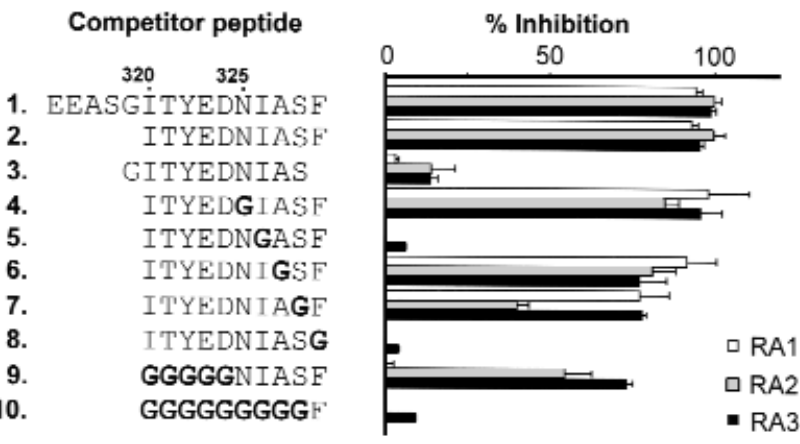

Figure 2

Competitive ELISA for fine epitope analysis. ELISA was performed with sera from three patients with RA (RA1, RA2, and RA3) whose titers of $\alpha$-RAPC 15 Ab's were $100,37.2$, and $9.6 \mathrm{U} / \mathrm{mL}$, respectively. Competitor peptide 1 is control peptide RAPC15. Peptides with 10 -amino acid sequences in RAPC15 where isoleucine 326 or $\mathrm{COOH}$-terminal phenylalanine 329 was replaced with glycine or removed (peptides 3,5 , and 8 ) exhibited no significant inhibition (< 25\%). Glycine 9 mer bearing $\mathrm{COOH}$-terminal phenylalanine (peptide 10) showed little inhibition. Percent inhibition values were calculated with the formula: (OD [nonabsorbed sera] - OD [sera absorbed by competitor peptides]) / OD [nonabsorbed sera] $\times 100$. 


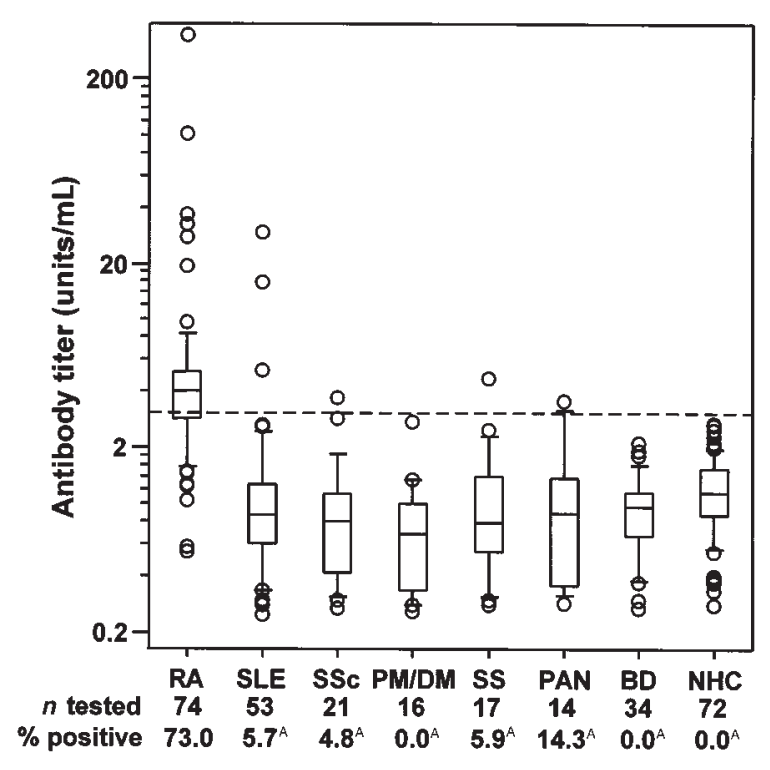

Figure 3

Serum titers of IgG-class $\alpha$-RAPC 15 Ab's in systemic rheumatic diseases. Positive titers (above the dashed line) were detected frequently in RA and occasionally in other diseases or healthy controls (SLE, systemic lupus erythematosus; SSc, systemic sclerosis; PM/DM, polymyositis/dermatomyositis; SS, Sjögren's syndrome; PAN, polyarteritis nodosa; BD, Behçet's disease; NHC, normal healthy control). The dashed line shows the cut-off value $(2.95 \mathrm{U} / \mathrm{mL})$ for positive titer of $\alpha$-RAPC15 Ab's (see the text). Horizontal bars represent 10, 25, 50 (median), 75, and 90 percentiles. Values outside of the $10-90$ percentile range are plotted by dots. ASignificantly lower than RA $\left(P<0.0001, \chi^{2}\right.$ test $)$.

RAPC15 in patients with systemic rheumatic diseases and healthy subjects, we assayed their sera by ELISA with RAPC15. The serum IgG-class antibody level (mean $\pm \mathrm{SD})$ was higher in $\mathrm{RA}(10.1 \pm 40.6 \mathrm{U} / \mathrm{mL})$ than in the controls $(1.25 \pm 0.56 \mathrm{U} / \mathrm{mL})$ and any other groups of systemic rheumatic diseases including systemic lupus erythematosus $(1.87 \pm 4.49 \mathrm{U} / \mathrm{mL})$, systemic sclerosis $(0.97 \pm 0.85 \mathrm{U} / \mathrm{mL})$, polymyositis/dermatomyositis $(0.81 \pm 0.63 \mathrm{U} / \mathrm{mL})$, Sjögren's syndrome $(1.13 \pm 1.10 \mathrm{U} / \mathrm{mL})$, polyarteritis nodosa $(1.24 \pm 1.05$ $\mathrm{U} / \mathrm{mL}$ ), and Behçet's disease $(0.97 \pm 0.46 \mathrm{U} / \mathrm{mL}$ ) (Figure 3 ). Using the mean $+3 \mathrm{SD}$ value in the control subjects as the cut-off value for $\alpha$-RAPC15 Ab's, the prevalence of $\alpha$-RAPC15 Ab's in RA became as much as $73 \%$ (Figure 3 ). This percentage was significantly larger than that in any disease group tested $(P<$ $0.0001, \chi^{2}$ test). In RA, CRP, erythrocyte sedimentation rate (ESR), peripheral blood platelet counts, and serum concentration of IL- 6 were significantly more elevated in $\alpha$-RAPC15 Ab-positive patients than in -negative ones (Table 1). Besides, $\alpha$-RAPC15 Ab titers were significantly correlated with CRP, ESR, and IL-6 concentration (Table 2). These results indicated that the presence of $\alpha$-RAPC15 Ab's was related with active states of RA. To elucidate what disease phase $\alpha$ RAPC15 Ab's reflect, we are investigating their time course of appearance.
Detection of gp130-RAPS molecules in synovial fluids and plasma. In synovial fluids and plasma from patients with RA and from control subjects, gp130-RAPS with a molecular weight of $50 \mathrm{kDa}$ was actually detected, as well as known long forms of 90 - and $110-\mathrm{kDa}$ sgp130 (Figure 4). gp130-RAPS was present in samples from patients with osteoarthritis and in healthy controls and was not specific to patients with RA.

Inhibitory function of gp130-RAPS against IL-6 and its interference by $\alpha$-RAPC15 of Ab's from patients with $R A$. To examine the effect of gp130-RAPS on IL-6 activity and the interaction with $\alpha$-RAPC15 Ab's, we assayed fibrinogen production in Hep G2 cells. gp130-RAPS interfered with IL-6-induced fibrinogen production in a dose-dependent manner (Figure $5 a)$. Affinity-purified $\alpha$-RAPC15 Ab's of a rabbit and RA patients dose-dependently elevated fibrinogen production in the presence of IL- 6 and gp130-RAPS (Figure 5b). Such effects of gp130-RAPS and $\alpha$ RAPC15 Ab's were observed as well in the cell proliferation assay using the IL-6-dependent murine hybridoma clone, MH60 (22) (data not shown). Thus, $\alpha$-RAPC15 Ab's actually associated with gP130-RAPS to block its IL-6-inhibitory function.

\section{Discussion}

gp130-RAPS, a novel soluble form of gp130, is a new autoantigen in RA. Its autoantibodies, $\alpha$-RAPC15 Ab's, are produced specifically and in association with disease activity in RA. Furthermore, $\alpha$-RAPC15 Ab's from patients with RA neutralized the inhibitory function of gp130-RAPS against IL-6. Thus, the $\alpha$-RAPC15 Ab can not only serve as a clinical indicator, but also provide an important clue to the pathology of RA.

Our cloned gp130-RAPS cDNA had an 83-bp fragment of gp130 cDNA missing. Cloning artifacts of a partial sequence deletion can originate when hairpin structure-forming mRNA templates are skipped during RT (23). Genomic gp130 DNA, however, had the introns adjacent to both the $5^{\prime}$ and $3^{\prime}$ ends of the deleted 83-bp fragment, which had a donor-acceptor structure compatible with the GT-AG rule. This shows that the 83-bp fragment is an exon and that mRNA splicing with loss of the entire fragment containing such introns and the 83-bp exon can produce gp130-RAPS mRNA. In addition, gp130-RAPS mRNA was actually

\section{Table 2}

Correlation of anti-RAPC15 Ab titers with disease activity parameters in patients with RA

\begin{tabular}{lccccc} 
& \multicolumn{3}{c}{ Pearson's correlation ${ }^{\mathrm{A}}$} & \multicolumn{2}{c}{ Spearman's rank correlation } \\
Parameter & $n$ & $r$ & $P$ & $r_{\mathrm{s}}$ & $P$ \\
CRP & 74 & 0.244 & $<0.05$ & $0.453<0.0001$ \\
ESR & 74 & 0.493 & $<0.0001$ & $0.459<0.0001$ \\
Platelets & 74 & 0.066 & NS & $0.333<0.005$ \\
IL-6 & 74 & 0.313 & $<0.01$ & $0.449<0.0001$ \\
RF & 71 & 0.028 & NS & $0.284<0.05$ \\
\hline
\end{tabular}

Comparison between ${ }^{\mathrm{A}}$ parametric and ${ }^{\mathrm{B}}$ nonparametric tests. 
Figure 4

Detection of gp130-RAPS in synovial fluids and plasma. Top: gp130-RAPS was immunoprecipitated (IP) with GPX22 mouse mAb to gP130 (GPX22 $\mathrm{mAb}$ ) and detected by Western blotting (Blot) with rabbit $\alpha$-RAPC15 Ab. Extra bands with a smaller molecular weight were probably formed by partially degraded molecules. Bottom: Besides gp130-RAPS, 90- and 110-kDa soluble forms of gp130 (broad fused bands) were immunoprecipitated with GPX22 $\mathrm{mAb}$ and detected by goat polyclonal anti-gp130 Ab ( $\alpha$-gp130 Ab). Control samples (lanes $\mathrm{C}$ ) were COS-7 cell-expressed recombinant gp130RAPS. OA, osteoarthritis.

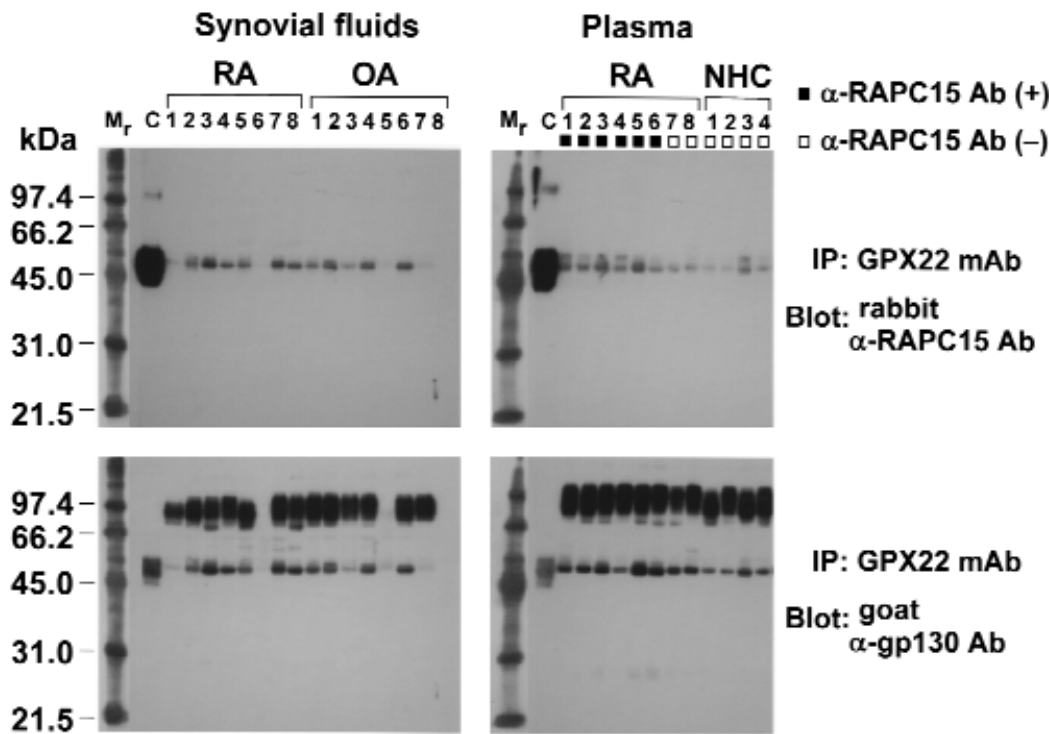

identified by RT-PCR in human cells. gp130-RAPS cDNA-transfected COS-7 cells produced its translated products. gp130-RAPS protein with a predicted molecular weight of $50 \mathrm{kDa}$ was actually found in synovial fluids and plasma by different specific Ab's. On the basis of these analyses of DNA, mRNA, and protein levels, we can conclude that gp130-RAPS is not a cloning artifact, but a splicing variant of gp130.

sgp130s are supposed to be generated by two mechanisms, proteolytic cleavage (shedding) and alternative mRNA splicing (24), although cDNA of sgp130 had

a

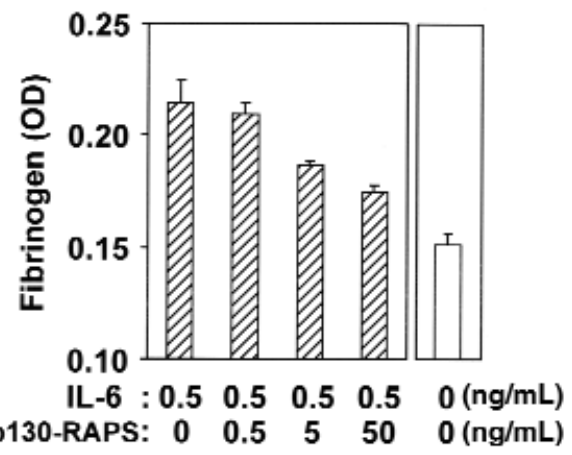

b

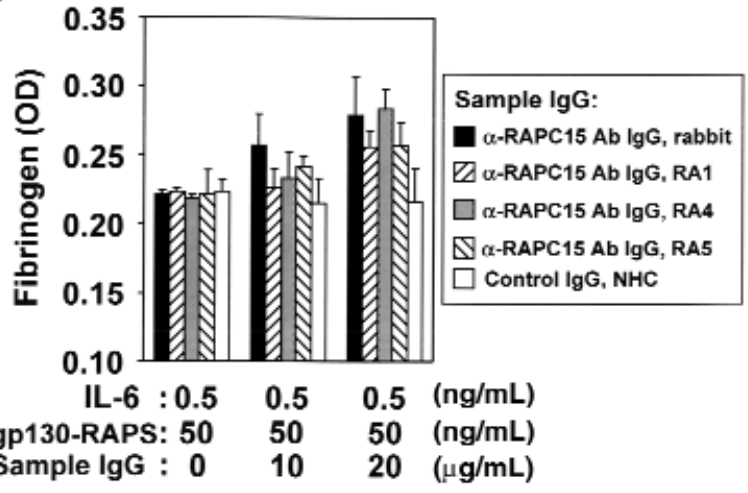

not been isolated. We succeeded in the cloning of sgp130 cDNA. sgp130s have been identified as three different molecules of about 50,90 , and $110 \mathrm{kDa}(10$, 25). Considering the similarity of molecular size, it is possible that gp130-RAPS is identical to the $50-\mathrm{kDa}$ species, which have not been cloned but whose existence was described while this study was under way (25). IL-6 upregulated the expression of gp130-RAPS in SF-1 cells. This suggests that gp130-RAPS production is a negative feedback to IL-6 stimulation.

In the Hep $\mathrm{G} 2$ fibrinogen production assay, rabbit $\alpha$ RAPC15 Ab, raised by immunization of RAPC15 peptides and affinity purified, inhibited the function of gp130-RAPS. Similarly, affinity-purified $\alpha$-RAPC15 Ab's from patients with RA impaired gp130-RAPS activity. These results show that $\alpha$-RAPC15 Ab's actually associate with native forms of gp130-RAPS molecules and exert a blocking effect. Therefore, $\alpha$-RAPC15 Ab's from patients with RA can be considered autoantibodies to gp130-RAPS. It is not clear why the same amounts of affinity-purified $\alpha$-RAPC15 Ab's had different activities for gp130-RAPS. It is probably due to the difference in the antibody reactivity to RAPC15 peptides covalently conjugated with agarose beads, to those on ELISA plates, and to the native form of gp130-RAPS. In fact, in West-

\footnotetext{
Figure 5

Inhibition of IL- 6 activity by gp130-RAPS (a) and reverse effect of $\alpha$ RAPC15 Ab's on its inhibition (b). Hep G2 cells were cultured in triplicate with test samples for 24 hours. (a) gp130-RAPS reduced IL-6-induced fibrinogen production by inhibiting IL-6 activity $(P<$ 0.05 , one-factor ANOVA). (b) $\alpha$-RAPC15 Ab's derived from a rabbit and patients with RA elevated fibrinogen production by neutralizing gp 130-RAPS and recovering IL- 6 activity $(P<0.05$ in rabbit and RA5; $P<0.005$ in RA4, one-factor ANOVA). IgG from normal healthy control (NHC) was the negative control. Titers per weight of $\alpha$-RAPC15 Ab's from patient RA1, RA4, and RA5 were 1.91, 1.03 and $0.16 \mathrm{U} / \mu \mathrm{g}$, respectively.
} 
ern blot analyses, affinity-purified $\alpha$-RAPC15 Ab's from patients with RA did not react with eukaryotic gp130RAPS molecules bound to membranes after SDS-PAGE, but reacted with prokaryotic ones (data not shown). In any case, $\alpha$-RAPC15 Ab's, antibodies to plate-attached RAPC15 peptides, seem to have biologic activity for gP130-RAPS under physiological conditions.

It was revealed that signals of gp130-related cytokines are transduced by dimerization of the cytoplasmic domains of gp130 after the coupling of two ligand-receptor complexes consisting of a ligand, its receptor, and gp130 (26). The known 90- and 110-kDa sgp130s are thought to interfere with the ligand signaling by forming nonfunctional ligand-receptor complexes due to a lack of cytoplasmic domains. gp130-RAPS appears to inhibit IL-6 activity in the same manner. $\alpha$-RAPC15 Ab's, therefore, seem to recover IL- 6 activity by disturbing such nonfunctional ligand-receptor complex formation.

$\alpha$-RAPC15 Ab's, neutralizing antibodies to gp130RAPS, promote IL-6 activity whereby gp130-RAPS is induced by negative feedback to IL-6. It remains to be clarified whether this phenomenon has significance in vivo and whether $\alpha$-RAPC15 Ab production is a cause or result in RA. It is well documented that some autoantibodies are pathogenic, modifying or inhibiting the activities of their target molecules in autoimmune and malignant diseases, e.g., autoantibody to $\beta 2$-glycoprotein-I in antiphospholipid syndrome, autoantibody to thyroid-stimulating hormone receptor in Graves disease, and autoantibody to acetylcholine receptor in myasthenia gravis. In RA, blocking autoantibody to calpastatin was reported to aid calpain activity in destructive joint inflammation $(27,28)$. Autoantibody to follistatin-related protein, which we reported previously (3), is another candidate and under investigation. Given its IL-6-supporting effect, and high prevalence and correlation with disease activity in RA, $\alpha$-RAPC15 Ab, autoantibody to gp130-RAPS, seems to play an important role in disease development in RA.

Detection of $\alpha$-RAPC15 Ab's may well be a more valuable clinical finding than known clinical parameters in RA because it has high sensitivity and specificity $(73.0 \%$ and $96.9 \%$, respectively, in Figure 3 ) and also reflects disease activity as already described here. In addition, ELISA with RAPC15 peptides is a promising tool for the detection of $\alpha$-RAPC15 Ab's, because of its simplicity and reproducibility due to the usage of synthetic peptides, rather than recombinant proteins or tissue extracts.

Why do $\alpha$-RAPC15 Ab's appear in patients with RA? One possibility is that $\alpha$-RAPC15 Ab's are generated directly against gP130-RAPS that is overexpressed in pathological states. Another possibility is that $\alpha$ RAPC15 Ab's are originally produced against infectious agents containing an NIASF or NIASF-like sequence and then cross-react to gp130-RAPS. The latter speculation seems more plausible than the former because the upregulated expression of gp130-RAPS was not specific to RA and observed in synovial fluids from patients with osteoarthritis as well as those with RA. In the pathogenesis of RA, infection with various organisms including viruses, bacteria, and mycoplasmas has been suggested to be the trigger of disease development (1). By using the FASTA program on the GenomeNet service (29), we found NIASF or NIASF-like sequences in several organic proteins; -NIASF- in human monocyte/macrophage serine esterase (A49816), yeast glutamate dehydrogenase (A25275), simian rotavirus SA11 glycoprotein VP7 precursor (VGXR1S), Streptococcus pyogenes exotoxin type B precursor (A37768), Escherichia coli dnaC protein (XMECNC), Haemophilus influenzae probable membrane protein HI0608 (I64080), Bacillus subtilis cheV protein (A55592); -NI-SF- in saimiriine herpesvirus 1 gene 34 protein (QQBEN3); -NI-F- in Escherichia coli ribonuclease T2 (S32940); and -I-SF- in Stapbylococcus aureus cell division protein FtsZ (S58814) (accession numbers of the PIR protein sequence database are in parentheses). Among them, we could not find any protein containing $\mathrm{COOH}$ terminal NIASF or NIASF-like sequences. However, interestingly, Streptococcus pyogenes, whose exotoxin precursor contains the NIASF sequence, has been reported to induce arthritis both in humans (30) and in an animal model (31). To identify pathogenic molecules in RA, $\alpha$ RAPC15 Ab's and synovial fluid and/or sera from patients with recent-onset RA may be useful materials as probes and antigen sources.

The $\alpha$-RAPC15 Ab, the autoantibody to gp130-RAPS, provides a novel parameter for the clinical characterization of RA. However, more intensive studies including follow-up surveys about the correlation between its titers and clinical manifestations are necessary to prove its advantages over available clinical tests and to establish its definitive significance in RA. In any case, gp130RAPS and its autoantibody may provide the tools with which to elucidate the pathogenesis of and to create new therapies for RA.

\section{Acknowledgments}

We thank T. Nakamura (Kyoto University) and colleagues for supplying the synovial tissue samples. We also thank T. Taga for technical advice and critical reading of the manuscript. This work was supported by Grants-in-Aid (no. 08457152) from the Ministry of Education, Science, and Culture of Japan, and by a grant from Funds for Comprehensive Research on Long-Term Chronic Disease from the Ministry of Health and Welfare of Japan.

\footnotetext{
1. Firestein, G.S. 1997. Etiology and pathogenesis of rheumatoid arthritis. In Textbook of rheumatology. Volume 1. W.N. Kelley, E.D. Harris, S. Ruddy, and C.B. Sledge, editors. W.B. Saunders Co. Philadelphia, Pennsylvania, USA. 851-888.

2. Tighe, H., and Carson, D.A. 1997. Rheumatoid factors. In Textbook of rheumatology. Volume 1. W.N. Kelley, E.D. Harris, S. Ruddy, and C.B. Sledge, editors. W.B. Saunders Co. Philadelphia, Pennsylvania, USA. 241-247.

3. Tanaka, M., et al. 1998. Cloning of follistatin-related protein as a novel autoantigen in systemic rheumatic diseases. Int. Immunol. 10:1305-1314.

4. Taga, T., et al. 1989. Interleukin- 6 triggers the association of its receptor with a possible signal transducer, gp130. Cell. 58:573-581.

5. Ip, N.Y., et al. 1992. CNTF and LIF act on neuronal cells via shared signaling pathways that involve the IL-6 signal transducing receptor com-
} 
ponent gp130. Cell. 69:1121-1132.

6. Gearing, D.P., et al. 1992. The IL-6 signal transducer, gp130: an oncostatin $\mathrm{M}$ receptor and affinity converter for the LIF receptor. Science. 255:1434-1437.

7. Liu, J., et al. 1992. Interleukin-6 signal transducer gp130 mediates oncostatin M signaling. J. Biol. Chem. 267:16763-16766.

8. Yin, T., et al. 1993. Involvement of IL-6 signal transducer gp130 in IL-11mediated signal transduction. J. Immunol. 151:2555-2561.

9. Pennica, D., et al. 1995. Expression cloning of cardiotrophin 1, a cytokine that induces cardiac myocyte hypertrophy. Proc. Natl. Acad. Sci. USA. 92:1142-1146

10. Narazaki, M., et al. 1993. Soluble forms of the interleukin- 6 signal-transducing receptor component gp130 in human serum possessing a potential to inhibit signals through membrane-anchored gp130. Blood. 82:1120-1126.

11. Feldmann, M., et al. 1996. Role of cytokines in rheumatoid arthritis. In Annual review of immunology. Volume 14. W.E. Paul, C.G. Fathman, and H. Metzger, editors. Annual Review Inc. Palo Alto, California, USA. 397-440.

12. Hirano, T. 1994. Interleukin-6. In The cytokine handbook. A.W. Thomson, editor. Academic Press Ltd. London, United Kingdom. 145-161.

13. Mihara, M., Moriya, Y., Kishimoto, T., and Ohsugi, Y. 1995. Interleukin6 (IL-6) induces the proliferation of synovial fibroblastic cells in the presence of soluble IL-6 receptor. Br. J. Rheumatol. 34:321-325.

14. Kotake, S., et al. 1996. Interleukin- 6 and soluble interleukin- 6 receptors in the synovial fluids from rheumatoid arthritis patients are responsible for osteoclast-like cell formation. J. Bone Miner. Res. 11:88-95.

15. Houssiau, F.A., et al. 1988. Interleukin-6 in synovial fluid and serum of patients with rheumatoid arthritis and other inflammatory arthritides. Arthritis Rheum. 31:784-788.

16. Wendling, D., Racadot, E., and Wijdenes, J. 1993. Treatment of severe rheumatoid arthritis by anti-interleukin 6 monoclonal antibody. $J$. Rheumatol. 20:259-262.

17. Yoshizaki, K., Nishimoto, N., Mihara, M., and Kishimoto, T. 1998. Therapy of rheumatoid arthritis by blocking IL-6 signal transduction with a humanized anti-IL-6 receptor antibody. Springer Semin. Immunopathol. 20:247-259.

18. Hibi, M., et al. 1990. Molecular cloning and expression of an IL-6 signal transducer, gp130. Cell. 63:1149-1157.

19. Saito, M., Yoshida, K., Hibi, M., Taga, T., and Kishimoto, T. 1992. Mole- cular cloning of a murine IL-6 receptor-associated signal transducer, gp130, and its regulated expression in vivo. J. Immunol. 148:4066-4071.

20. Taga, T. et al. 1992. Functional inhibition of hematopoietic and neurotrophic cytokines by blocking the interleukin 6 signal transducer gp130. Proc. Natl. Acad. Sci. USA. 89:10998-11001.

21. Gauldie, J., Richards, C. Harnish, D., Lansdorp, P, and Baumann, $H$. 1987. Interferon beta 2/B-cell stimulatory factor type 2 shares identity with monocyte-derived hepatocyte-stimulating factor and regulates the major acute phase protein response in liver cells. Proc. Natl. Acad. Sci. USA. 84:7251-7255.

22. Matsuda, T., Hirano, T., and Kishimoto, T. 1988. Establishment of an interleukin 6 (IL 6)/B cell stimulatory factor 2-dependent cell line and preparation of anti-IL 6 monoclonal antibodies. Eur. J. Immunol. 18:951-956.

23. Spring, D.J., and Krebs, E.G. 1999. Deletion of 11 amino acids in p90(rskmo-1) abolishes kinase activity. Mol. Cell. Biol. 19:317-320.

24. Diamant, M., et al. 1997. Cloning and expression of an alternatively spliced mRNA encoding a soluble form of the human interleukin- 6 signal transducer gp130. FEBS Lett. 412:379-384.

25. Zhang, J.G., et al. 1998. Identification and characterization of two distinct truncated forms of gp130 and a soluble form of leukemia inhibitory factor receptor alpha-chain in normal human urine and plasma. J. Biol. Chem. 273:10798-10805.

26. Paonessa, G., et al. 1995. Two distinct and independent sites on IL-6 trigger gp 130 dimer formation and signalling. EMBO J. 14:1942-1951.

27. Despres, N., Talbot, G., Plouffe, B., Boire, G., and Menard, H.A. 1995. Detection and expression of a cDNA clone that encodes a polypeptide containing two inhibitory domains of human calpastatin and its recognition by rheumatoid arthritis sera. J. Clin. Invest. 95:1891-1896.

28. Mimori, T., et al. 1995. Autoantibodies to calpastatin (an endogenous inhibitor for calcium-dependent neutral protease, calpain) in systemic rheumatic diseases. Proc. Natl. Acad. Sci. USA. 92:7267-7271.

29. Genome Net WWW Server. http://www.genome.ad.jp. Accessed May 12, 2000

30. Bisno, A.L. 1997. Rheumatic fever. In Textbook of rheumatology. Volume 2. W.N. Kelley, E.D. Harris, S. Ruddy, and C.B. Sledge, editors. W.B. Saunders Co. Philadelphia, Pennsylvania, USA. 1225-1238.

31. Sternberg, E.M., et al. 1989. Inflammatory mediator-induced hypothalamic-pituitary-adrenal axis activation is defective in streptococcal cell wall arthritis-susceptible Lewis rats. Proc. Natl. Acad. Sci. USA. 86:2374-2378. 